\title{
DELAYED REMISSION AFTER THYMECTOMY FOR MYASTHENIA GRAVIS OF THE PURELY OCULAR TYPE
}

Hiroshige Nakamura, MD

Yuji Taniguchi, MD

Yoshimasa Suzuki, MD

Yoshiyuki Tanaka, MD

Kiyosuke Ishiguro, MD

Mikihisa Fukuda, MD

Hiroshi Hara, MD

Tohru Mori, MD
Twenty-two cases of purely ocular myasthenia gravis were reviewed to evaluate the long-term effects of thymectomy. Remission rate increased gradually with time (11.8\% at 3 years, $23.1 \%$ at 5 years, and $33.3 \%$ at 10 years). Analysis of factors influencing remission with time showed that patients with short duration of illness attained remission significantly earlier ( $p=0.035$ at 5-year follow-up). One of 22 patients with purely ocular myasthenia gravis $(4.5 \%)$ had disease progression. Because ocular myasthenia gravis often progresses to the generalized type and because duration of illness before operation is one of important factors influencing remission, we conclude that thymectomy in the earlier stages of the disease is the preferred treatment for ocular myasthenia gravis, just as for generalized myasthenia gravis. (J Thorac Cardiovasc Surg 1996;112:371-5)
$\mathrm{T}$ hymectomy has been established as an effective therapeutic modality for generalized myasthenia gravis (MG) ${ }^{1,2}$ Recently, the trend has been toward more extensive to complete dissection of thymic tissues $^{3}$; however, for MG of the ocular type the effectiveness of thymectomy remains controversial. Because ocular MG is not a life-threatening disease and may also have natural remission, thymectomy for ocular MG does not have as great an effect on it as on generalized MG. On the other hand, ocular MG often progresses to generalized MG and may be complicated by occult thymoma not detected before operation.

For these reasons, we have treated ocular MG with thymectomy. To evaluate the effect of thymectomy and analyze the factors influencing outcome, we performed a retrospective review of 22 cases of purely ocular MG treated with thymectomy.

\section{Patients and methods}

The cases of 22 consecutive patients with ocular MG without thymoma who underwent thymectomy at Tottori University Hospital between January 1971 and December

From the Second Department of Surgery, Tottori University Faculty of Medicine, Yonago, Japan.

Received for publication Jan. 17, 1995; revisions requested April 4, 1995; revisions received Jan. 9, 1996; accepted for publication Jan. 9, 1996.

Address for reprints: Hiroshige Nakamura, MD, Second Department of Surgery, Tottori University Faculty of Medicine, 36-1 Nishi-Machi Yonago-City, 683, Tottori, Japan.

Copyright $(\subset) 1996$ by Mosby-Year Book, Inc.

$0022-5223 / 96 \$ 5.00+0 \quad \mathbf{1 2} / \mathbf{1} / 7 \mathbf{7 1 7 2 3}$
1993 were reviewed. These were eight male and 14 female patients ranging in age from 9 to 60 years (mean 34.3 years). Before operation, Grave's disease was detected in four patients and Hashimoto's disease was detected in one patient. Both thyroid diseases were diagnosed by abnormal serum levels of thyroid hormones and autoantibodies (anti-thyroid stimulating hormone receptor, antithyroglobulin, and antimicrosomal antibodies). All patients were initially evaluated by neurologists and determined to have ocular MG (fluctuating weakness of ocular muscles only) by neurologic examination. A positive result of edrophonium testing and waning phenomenon of only orbicularis oculi muscle on electromyography were the most important findings for all patients in the diagnosis of ocular MG. Serum titers of anti-acetylcholine receptor antibody (anti-AchR Ab) were measured in 18 of the 22 patients by immunoprecipitation iodine 125-labeled $\alpha$-bungarotoxin-acetylcholine receptor complex (normal level is less than $0.16 \mathrm{pmol} / \mathrm{ml}$ ). Greater than normal levels of anti-AchR $\mathrm{Ab}$ were not always necessary for diagnosis of ocular $\mathrm{MG}$.

For preoperative treatment, anticholinesterase agents (distigmine bromide or pyridostigmine bromide) were used in 16 cases, steroids were used in three cases, and nothing was used in three cases (Table I). The operative procedures were performed through the transsternal route. Simple thymectomy was performed in four cases before 1980; extended thymectomy, accompanied by total removal of the anterior mediastinal adipose tissue, which includes thymus tissues, was performed in 18 cases after 1981. This was established as a standard method. After the operation, in which steroid treatments were started for all patients in the early postoperative period, with pulsed therapy at the beginning and gradually decreasing drug doses later. Postoperative assessment of the effects of thymectomy on ocular MG was conducted at 3 months, 6 months, and $1,2,3,4,5,7$, and 10 years after operation. Mean follow-up time for all patients was 69.1 months. The classification system used to grade clinical improvement is 


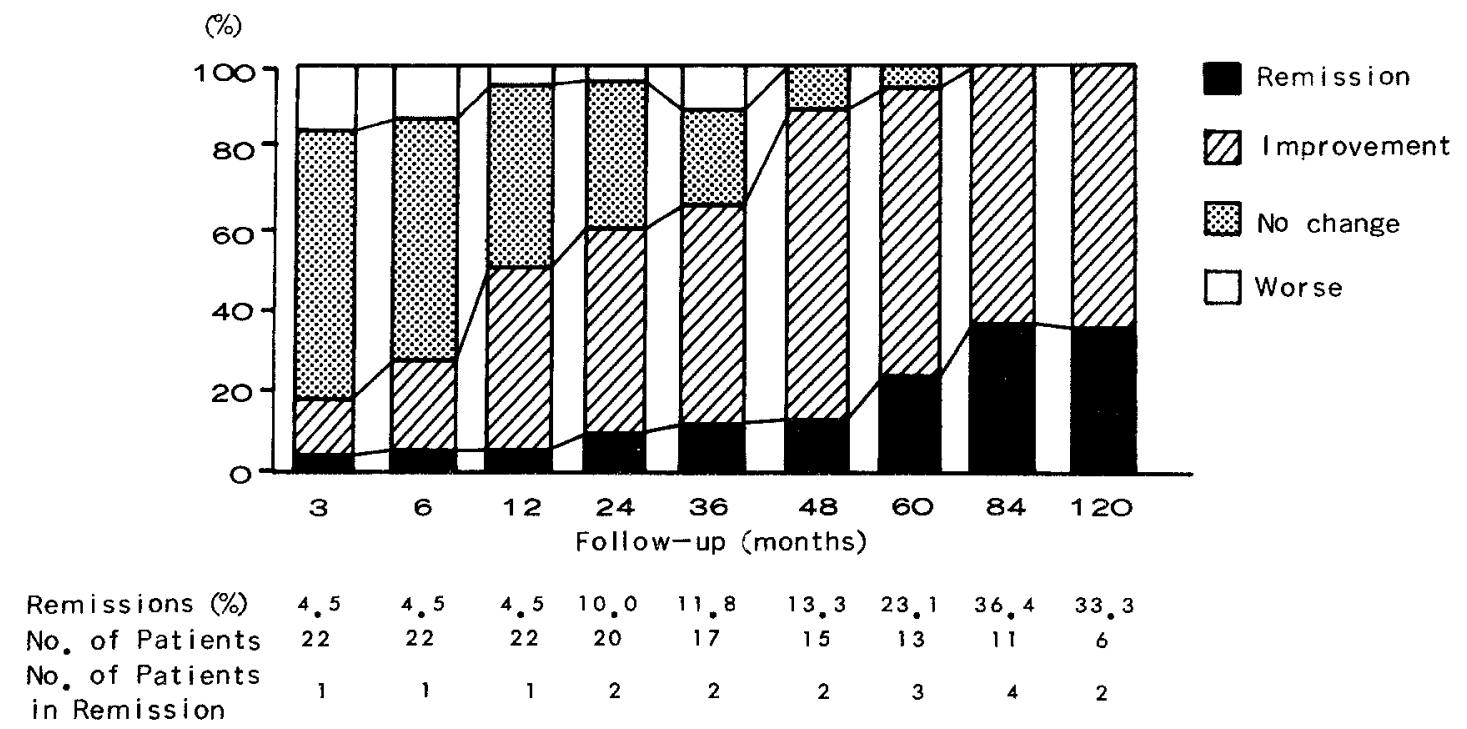

Fig. 1. The effects with time of 22 thymectomies for purely ocular MG.

Table I. Preoperative medications in ocular $M G$

\begin{tabular}{lc}
\hline \multicolumn{1}{c}{ Medication } & No. of cases \\
\hline Anticholinesterase agents (distigmine & 16 \\
bromide or pyridostigmine bromide) & \\
Steroids & 3 \\
Nothing & 3 \\
\hline
\end{tabular}

Table II. Classification of postoperative responses in ocular $M G$

\begin{tabular}{lll}
\hline \multicolumn{1}{c}{ Response } & Symptoms & $\begin{array}{l}\text { Medication } \\
\text { required }\end{array}$ \\
\hline Remission & None & None \\
Improvement & Improved & Decreased \\
& Improved & Unchanged \\
& Unchanged & Decreased \\
No change & Unchanged & Unchanged \\
& Improved & Increased \\
Worse & Worse & Increased \\
& Worse & Unchanged \\
& Unchanged & Increased \\
Death & & \\
\hline
\end{tabular}

shown in Table II. Remission was defined as complete freedom from symptoms without medication for longer than 3 months. Pathologic studies were performed by rereading the actual slide for this study, and hyperplasia of germinal center of thymic tissue was carefully examined.

The $\chi^{2}$ test or Fisher's Exact Test and the KruskalWallis nonparametric method were used for statistical analysis of differences. A $p$ value of less than 0.05 was considered statistically significant.
Table III. Results of thymectomy for ocular $M G$

\begin{tabular}{lll}
\hline & \multicolumn{2}{c}{ Remission } \\
\cline { 2 - 3 } & No. & $\%$ \\
\hline Total & $5 / 22$ & 22.7 \\
$\quad$ Age (yr) & & \\
$\quad \leq 39$ & $4 / 17$ & 23.5 \\
$\quad \geq 40$ & $1 / 5$ & 20.0 \\
Age of onset (yr) & & \\
$\quad \leq 34$ & $4 / 15$ & 26.7 \\
$\quad \geq 35$ & $1 / 7$ & 14.3 \\
Duration of illness (mo) & & \\
$\quad \leq 12$ & $3 / 8$ & 37.5 \\
$\quad \geq 13$ & $2 / 14$ & 14.3 \\
Sex & & \\
$\quad$ Male & $2 / 8$ & 25.0 \\
$\quad$ Female & $3 / 14$ & 21.4 \\
Operative procedure & & \\
$\quad$ Extended thymectomy & $4 / 18$ & 22.2 \\
$\quad$ Thymectomy & $1 / 4$ & 25.0 \\
Germinal center & & \\
$\quad$ Positive & $4 / 14$ & 28.6 \\
$\quad$ Negative & $1 / 8$ & 12.5 \\
Anti-AchR Ab & & \\
$\quad$ Positive & $2 / 8$ & 25.0 \\
$\quad$ Negative & $2 / 10$ & 20.0 \\
\hline
\end{tabular}

Remission was evaluated at the last observation point in each case.

\section{Results}

For purely ocular MG without thymoma, remission rate was $22.7 \%$ at the last observation point. Analysis of factors influencing remission at the last observation point showed no statistically significant differences between all pairs of groups by age, age of 

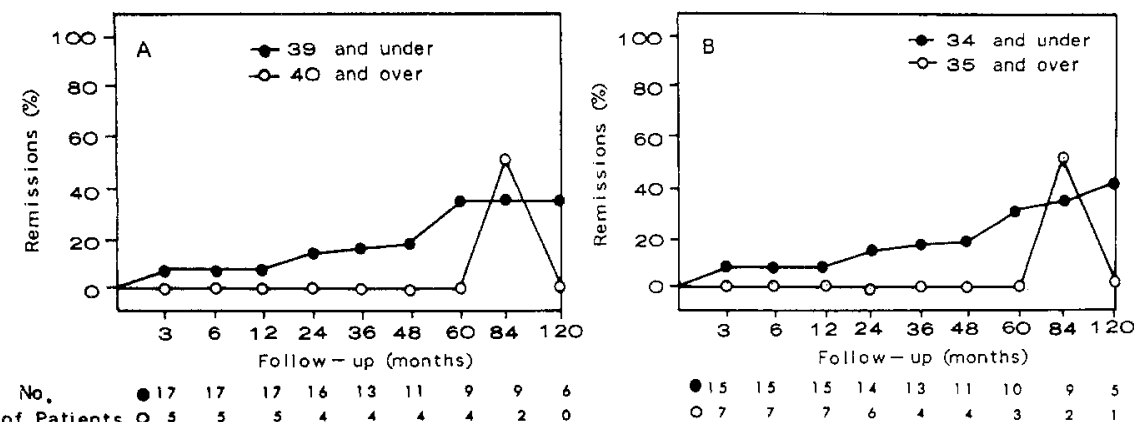

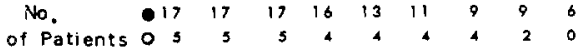
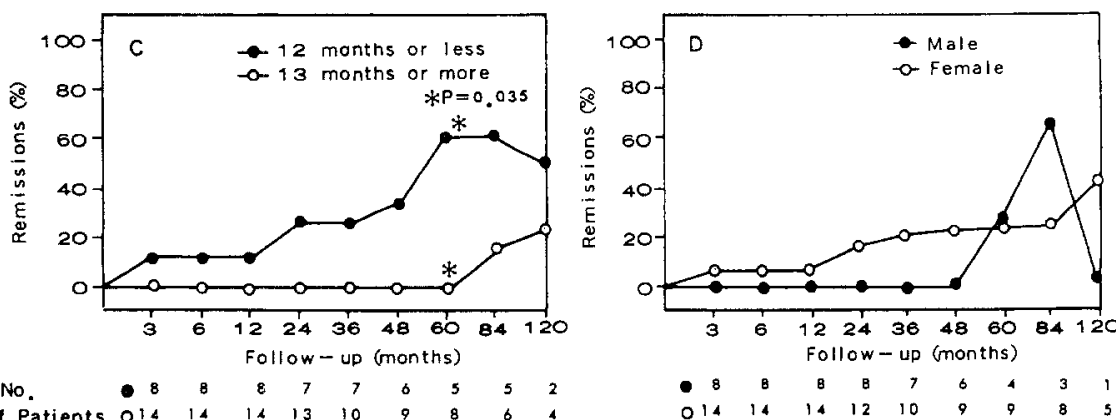

No. $\begin{array}{lllllllll}0 & 8 & 8 & 8 & 7 & 7 & 6 & 5 & 5 \\ 014 & 14 & 14 & 13 & 10 & 9 & 8 & 6\end{array}$
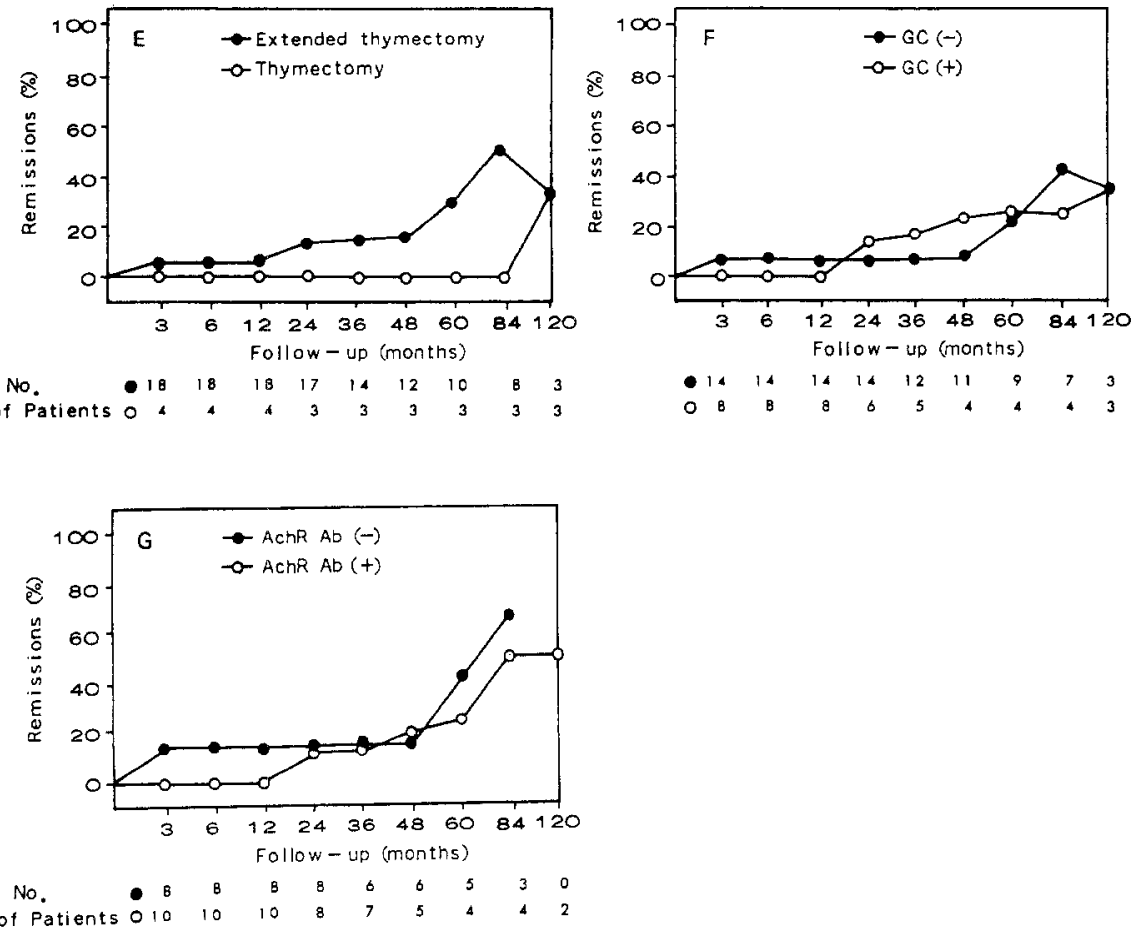

Fig. 2. Remission rate after thymectomy for purely ocular MG over time according to age (A), age of onset (B), duration of illness before thymectomy (C), sex (D), operative procedure (E), germinal center $(G C, \mathbf{F})$, and anti-AchR Ab (G). At the 5-year follow up interval, patients with duration of illness of 12 months or less had significantly better remission than those with durations of 13 months or more ( $p=0.035$ by Fisher's Exact Test). 


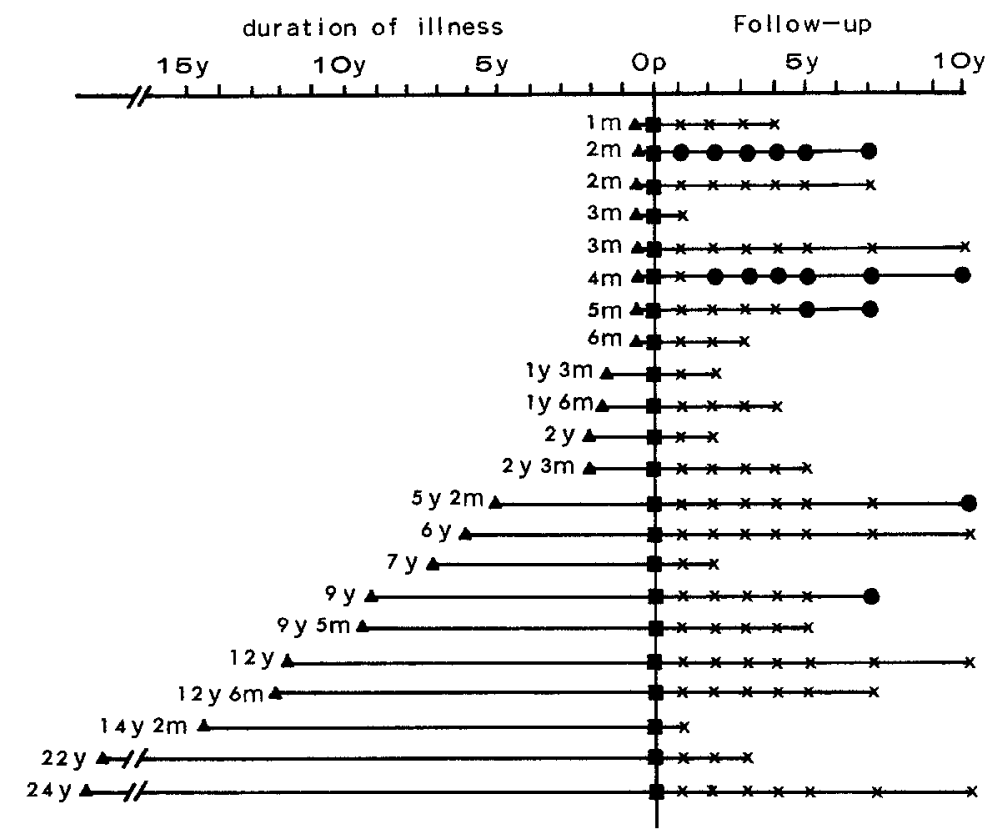

Fig. 3. Relationship between remission and duration of illness before thymectomy in all 22 patients with ocular MG. Patients with shorter duration of illness had a tendency toward earlier remission. $O P$, Operation; $\mathbf{\Lambda}$, onset of disease; $\mathbf{\square}$, operation; $\boldsymbol{\bullet}$, remission; $\mathrm{x}$, no remission.

Table IV. Remission rate related to $M G$ classification and the duration of illness

\begin{tabular}{lcccc}
\hline & & Duration of & \multicolumn{2}{c}{ Remission rate (\%) } \\
\cline { 3 - 5 } MG classification & Cases & illness (mo) & 10 years & Last observation \\
\hline I (Ocular) & 22 & 71.1 & 33.3 & 22.7 \\
IIa (Mild general) & 26 & 45.4 & 50.0 & 30.8 \\
IIb (Severe general) & 16 & 47.6 & 40.0 & 37.5 \\
\hline
\end{tabular}

Ocular MG showed a tendency toward longer durations of illness and worse remission rates than seen in the other two groups.

onset, duration of illness, sex, operative procedure, hyperplasia of germinal center, and anti-AchR $\mathrm{Ab}$ (Table III). When the postoperative time course was studied, remission rates were seen to increase gradually with time $(4.5 \%$ at 1 year, $11.8 \%$ at 3 years, $23.1 \%$ at 5 years, $33.3 \%$ at 10 years; Fig. 1). Analysis of factors influencing remission showed that duration of illness before operation was an important factor in the postoperative time course study. Cases in which the duration of illness before operation was less than 12 months showed significant earlier and better remission than those with duration longer than 12 months before operation $(p=0.035$ at 5-year follow-up; Figs. 2 and 3).

Disease progression of purely ocular MG occurred in one of 22 patients (4.5\%), a 34-year-old woman whose disease progressed to the generalized type of MG shortly after operation. There was no morbidity associated with the surgical procedure and no deaths occurred during hospitalization.

Results from patients with ocular MG were compared with those of patients with the other types of MG without thymoma who underwent operation during the same period. Patients with ocular MG had the longest durations of illness before operation and worst remission rates of the three groups (Table IV). There were, however, no statistically significant differences.

\section{Discussion}

Ocular MG is defined as a disease with only ocular symptoms and without abnormal general findings even on electromyography. An important question is whether ocular symptoms are the initial symptoms of generalized MG. Bever and colleagues $^{4}$ reported that $43(36 \%)$ of 119 cases of 
ocular MG progressed to the generalized type of MG within 2 years. In our study, only one of 22 patients $(4.5 \%)$ had disease progression to generalized $\mathrm{MG}$, a particularly favorable result compared with the previous study. It is still difficult, however, to anticipate which cases will progress to generalized MG. In the therapy of ocular MG, Fisher and Schwartmann ${ }^{5}$ reported that treatment with steroids alone was associated with relapse and that surgical treatments were indicated as for generalized MG. On the other hand, Oosterhuis ${ }^{6}$ reported that about $30 \%$ of cases of ocular MG underwent natural remission during the course of 15 years. These results caused confusion in devising a strategy for treatment of ocular MG.

Little published literature about the long-term results of thymectomy for ocular MG is available because of the small number of cases. Even in the large series of 2062 cases of all types of MG reported on by Papatestas and coworkers, ${ }^{7}$ thymectomy was performed in only $12(3.8 \%)$ of 313 cases of ocular MG. In their report, they showed that remission occurred in $59(20 \%)$ of 301 patients who did not undergo thymectomy and in two (17\%) of 12 patients who underwent thymectomy. These results suggested that it would not be helpful to perform surgical procedure in cases of ocular MG. Schumm and colleagues ${ }^{8}$ showed the effectiveness of thymectomy for ocular $\mathrm{MG}$, however, with a palliation rate of almost $80 \%$ and remission occurring in three (16.7\%) of 18 patients during a mean follow-up of only 26 months.

In the analysis of factors influencing remission of generalized MG, Papatestas and coworkers ${ }^{7}$ showed that severity of disease, presence of thymoma, duration of illness, and surgical method affected outcome, whereas First and associates ${ }^{9}$ demonstrated by multiple-regression analysis that age, sex, and preoperative stage were significant factors. Monden and coworkers, ${ }^{10}$ who found duration of illness before operation to be a significant factor in the outcome of generalized MG, showed a correlation between duration of illness before operation and thymic hyperplasia of the germinal center on histopathologic examination. In the future, indexes concerned with the progression of ocular to generalized MG may include factors such as duration of illness, thymic hyperplasia of the germinal center, and anti-AchR $\mathrm{Ab}$ titer.

Our study showed an effectiveness for thymectomy in the treatment purely ocular MG just as in the treatment of generalized $\mathrm{MG}$, especially for protection against progression to generalized $\mathrm{MG}$. It is also important that there is little risk in performing the surgical procedure and managing the perioperative period versus generalized MG. Some of these patients, however, might have had natural remission later without thymectomy. If the factors affecting progression to generalized $\mathrm{MG}$ can be defined, it will be possible to select patients to undergo thymectomy, but for now because one of important factors influencing remission of ocular MG is the duration of illness before thymectomy, we recommend thymectomy in the early stages of the disease as the preferred treatment in ocular MG, just as in generalized MG.

\section{REFERENCES}

1. Blalock A, Mason MF, Morgan HJ, Riven SS. Myasthenia gravis and tumors of the thymic region: report of case in which tumor was removed. Ann Surg 1939;110:544-61.

2. Papatestas AE, Alpert LI, Osserman KE, Osserman RS, Kark KE. Studies in myasthenia gravis: effects of thymectomy. Am J Med 1971;50:465-74.

3. Jaretzki A 3rd, Penn AS, Younger DS, et al. "Maximal" thymectomy for myasthenia gravis. J Thorac Cardiovasc Surg 1988;96:711-6.

4. Bever CT, Aquio AV, Penn AS, Lovelace RA, Rowland LP. Prognosis in ocular myasthenia (abstract). Neurology 1980; 30:387.

5. Fischer KC, Schwartmann RJ. Oral corticosteroids in the treatment of ocular myasthenia gravis. Ann N Y Acad Sci 1976;274:596-607.

6. Oosterhuis HJ. Observations of the natural history of myasthenia gravis and the effect of thymectomy. Ann N Y Acad Sci 1981;377:678-90.

7. Papatestas AE, Genkins G, Kornfeld P, Eisenkraft JB, Fagerstrom RP, Pozner J, et al. Effects of thymectomy in myasthenia gravis. Ann Surg 1987;206:79-88.

8. Schumm F, Wiethölter H, Fateh-Moghadam A, Dickgans J. Thymectomy in myasthenia with pure ocular symptoms. J Neurol Neurosurg Psychiatry 1985;48:332-7.

9. First WH, Thirumalai S, Doehring CB, et al. Thymectomy for the myasthenia gravis patients: factors influencing outcome. Ann Thorac Surg 1994;57:334-8.

10. Monden Y, Nakahara K, Kagotani K, Fujii Y, Nanjo S, Masoka A, et al. Effects of preoperative duration of symptoms on patients with myasthenia gravis. Ann Thorac Surg 1984;38:287-91. 